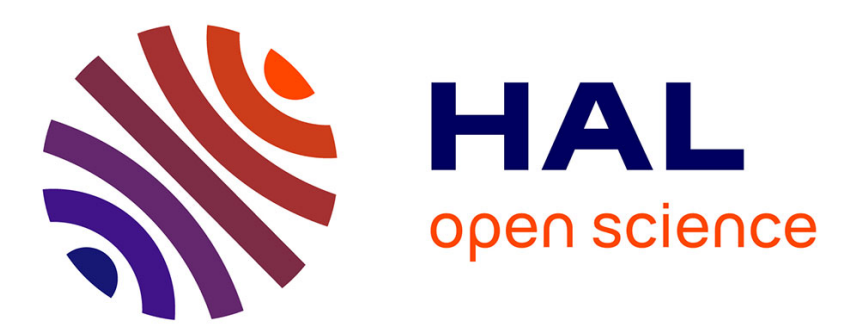

\title{
Twisting of pre-twinned $\alpha$-Fe nanowires: from mild to wild avalanche dynamics
}

Yang Yang, Suzhi Li, Xiangdong Ding, Jun Sun, Jérôme Weiss, Ekhard K.H. Salje

\section{- To cite this version:}

Yang Yang, Suzhi Li, Xiangdong Ding, Jun Sun, Jérôme Weiss, et al.. Twisting of pre-twinned $\alpha$-Fe nanowires: from mild to wild avalanche dynamics. Acta Materialia, 2020, 195, pp.50-58. 10.1016/j.actamat.2020.04.023 . hal-03001593

\section{HAL Id: hal-03001593 https://hal.science/hal-03001593}

Submitted on 27 Nov 2020

HAL is a multi-disciplinary open access archive for the deposit and dissemination of scientific research documents, whether they are published or not. The documents may come from teaching and research institutions in France or abroad, or from public or private research centers.
L'archive ouverte pluridisciplinaire HAL, est destinée au dépôt et à la diffusion de documents scientifiques de niveau recherche, publiés ou non, émanant des établissements d'enseignement et de recherche français ou étrangers, des laboratoires publics ou privés. 


\section{Twisting of pre-twinned $\alpha-F e$ nanowires: \\ from mild to wild avalanche dynamics}

Yang Yang ${ }^{a}$, Suzhi Li ${ }^{a,{ }^{*}}$, Xiangdong Ding ${ }^{a,{ }^{*}}$, Jun $\operatorname{Sun}^{a}$, Jerome Weiss ${ }^{b, *}$, Ekhard K.H. Salje $\mathrm{e}^{a, c,{ }^{*}}$

a. State Key Laboratory for Mechanical Behaviour of Materials, Xi'an Jiaotong University, Xi'an 710049, China

b. IsTerre, CNRS/Université Grenoble Alpes, 38401, Grenoble, France

c. Department of Earth Sciences, University of Cambridge, Cambridge CB2 3EQ, UK

* Authors to whom correspondence should be addressed:

lisuzhi@xjtu.edu.cn (S.L.)

dingxd@mail.xjtu.edu.cn (X.D.)

jerome.weiss@univ-grenoble-alpes.fr (J.W.)

ekhard@esc.cam.ac.uk (E.K.H.S.) 


\begin{abstract}
We studied the torsion behavior of $\alpha$-Fe nanowires seeded with twin boundaries (TBs) using molecular dynamics simulations. Twisting the wire generates topological defects in the twin walls, namely kinks inside the twin walls for small twist angles, and junctions between kinks for large twist angles. During twisting the kink motion is jerky and uncorrelated at small twist angles. The probability density function (PDF) of jerk energies follows approximately a Gaussian distribution, indicating a mild deformation mode. The kink dynamics transforms from mild to wild at larger twist angles when complex twin patterns with a high density of junctions are generated. The collective motion of kinks now shows avalanche behavior with the energy being power-law distributed. The wildness, which measures the proportion of strain energy relaxed through such avalanches, is correlated with the junction density, and controlled by the external length scale (wire diameter) as well as an internal length scale (twin boundary spacing). Good strain-stress recoverability is achieved when unloading the wire before the formation of complex twin patterns. We correlate the evolution of twin patterns with a statistical analysis of jerk dynamics, which identifies the unique mechanical properties governed by twin boundary motion in nanowires.
\end{abstract}

Keywords: twinning; $\alpha-\mathrm{Fe}$ nanowire; torsion; avalanches; mild wild fluctuations; torsion spectroscopy 


\section{Introduction}

In metals, plastic deformation is associated with the motion and interactions of structural defects such as dislocations or twin boundaries. The classical paradigm of dislocation-mediated plasticity in bulk crystals assumes that correlations between individual dislocation motions are weak, allowing a homogenized description. However, jerky, intermittent plasticity was already noticed by Orowan and Becker on Zinc rods in 1932 [1]. Twenty years ago, acoustic emission (AE) data in plastically deformed HCP materials showed that plastic jerks are, in these materials, clustered in space [2] and time [3], as well as power-law distributed in size and energy [4], in complete contradiction with the classical paradigm. It was realized more recently that a full spectrum of behavior can be observed: from wild plasticity accommodated essentially through dislocation avalanches, e.g. in HCP materials, to mild plasticity for which plastic jerks are uncorrelated and associated with a characteristic size or energy scale ("Gaussian-like"), e.g. in FCC metals at large systems sizes. Furthermore, mild and wild fluctuations can coexist during plastic deformation [5]. The wildness of dislocation-mediated plasticity is associated with size effects: In materials characterized by a mild plasticity at large scales, such as in FCC metals, deformation becomes wild for sub- $\mu$ m sample sizes $[6,7]$, signing a "smaller is wilder" size effect [8] with potential detrimental effects for micro/nanoscale engineering $[9,10]$. Wildness also depends on an internal length scale that emerges from dislocations with mutual interactions in pure metals. The length scale corresponds to a dislocation mean free path in some cases, or to added defects in alloys [8]. These two size effects combine through a unique dimensionless ratio of scales (external/internal) that controls the wild-to-mild transition. Thus, the wildness of dislocationmediated plasticity is not controlled by the system size only. Pre-straining the material before cutting the micropillar will develop an internal microstructure of dislocation topologies (at least in FCC materials) $[9,11,12]$, with a characteristic internal scale. This external/internal ratio controls wildness in dislocation-mediated plasticity [8].

In HCP metals like Zinc or Cadmium, plasticity occurs through a combination of dislocation motion and twinning events, with the latter dominating deformation at large strains. It was shown from a statistical analysis of AE data that the two mechanisms interact within an overall wild regime, meaning that dislocation avalanches can trigger twinning events, as well as the inverse effect of twins triggering dislocations [13]. However, work has been carried out rarely for twin-mediated deformation in systems where twin boundaries are tailored. Known as domain boundary engineering, the manipulation of twin patterns is an effective way for achieving unique properties in materials [14]. The fundamental idea is that domain boundaries possess electronic transport [15], polarization [16-18], heat conduction [19] and mechanical properties [20-27] that do not exist in the bulk. For example, the domain walls are observed to be polar while the bulk counterpart is non-polar in $\mathrm{CaTiO}_{3}$ [28]. The domain boundaries 
become superconducting in insulating $\mathrm{WO}_{3-\mathrm{x}}$ [29]. Twin boundaries (TBs) are found to greatly improve the strength and toughness in nano-twinned materials [23, 25, 26]. In addition, some functional properties can be achieved with the aid of TB motion, such as the recovery of large deformation [21], or the storage of mechanical energy [30]. These unique properties are strongly dependent on the complexity of twin patterns, however. Sometimes, the formation of a highly complex twin pattern could even degrade the functional properties [31].

Many studies have been carried out to determine the movement of domain walls under a uniform stress field, such as uniaxial tension or compression [32, 33]. However, little is known how twin boundaries evolve under non-uniform applied stress. Early work has shown that bending deformation could generate nonconventional $\{110\}$-type interfaces in $\alpha$-Fe nanowires[22]. Such interfaces provide the primary driving force for shape recovery upon unloading, leading to superelasticity. Besides bending, torsion is another typical deformation mode that generates shear stresses and strain gradients in devices such as nano-springs [34], torsional oscillators [35], torsional actuators [36]. As an example, six-fold twinned $\alpha$-Fe nanowires were found to sustain very large twists under torsion [31]. Yet, the structural evolution, especially the role that TBs play under twisting, is still not well understood.

In this paper, using molecular dynamics simulations, we study the mechanical performance of twinned $\alpha$-Fe nanowires during torsion and perform a statistical analysis of the evolution of twin walls and deformation jerks during deformation. We found that torsion promotes the deformation of TBs by kink nucleation and motion. The recoverability is directly related to the complexity of the twin patterns. For small-applied twist angles, the nanowires recover completely under unloading and only isolated kinks are generated. However, such recoverability is largely suppressed when a high density of junctions is formed through multikink intersections. Our statistical analysis indicates that the deformation mode changes from a mild regime at small twist angles to a wild regime at higher angles. In addition, the associated wildness is controlled by both an internal length scale (twin boundary spacing) and the external length scale (wire diameter, while keeping the twin boundary spacing constant), much like in dislocation-mediated plasticity [8, 37]. This suggests material design strategies to limit these detrimental wild fluctuations and the generation of complex patterns.

\section{Simulation method}

The embedded atom method [38] (EAM) developed by Mendelev [39] is used to describe the atomic interactions of $\alpha-\mathrm{Fe}$. This potential gives a good fit to the basic properties of $\alpha$-Fe, such as the elastic constants, interstitial and vacancy formation energy, six-fold core structure of screw dislocation, etc. We constructed [112]-oriented twinned nanowires with preexisting $\{112\} /<111>$ TBs, as shown in Fig. 1a. All nanowires have circular cross sections and maintain 
the same aspect ratio. The diameter $d$ varies from $5 \mathrm{~nm}$ to $15 \mathrm{~nm}$, with the total length varying from $22.5 \mathrm{~nm}$ to $67.5 \mathrm{~nm}$. The twin spacing $s$ ranges from $3.8 \mathrm{~nm}$ to $15.0 \mathrm{~nm}$. Before torsion, the nanowires were relaxed at $1 \mathrm{~K}$ for 0.5 nanoseconds using a Nosé-Hoover thermostat [40, 41]. Several atomic layers at both ends of the nanowire are fixed rigidly as the loading grip during torsion. We then rotate one rigid end along the axis of the nanowire while the other end is fixed (see the inset in Fig. 1a). The maximum shear strain $\gamma_{\max }$ is calculated as half of the torsion angle $\theta$ divided by the aspect ratio of the sample, $\gamma_{\max }=\theta d /(2 L)$. The maximum shear stress is $\tau_{\max }=T d /(2 I)$, where $T$ is the torque applied and $I$ is the polar moment of inertia. The twist was applied with a rate of $0.05^{\circ}$ per picosecond, corresponding to a shear strain-rate around $1 \times 10^{7} \mathrm{~s}^{-1}$. Unloading was performed in a similar way by rotating the grip in the opposite direction. The MD calculations were carried out in a canonical ensemble by using LAMMPS code [42] and the atomic configurations were displayed by AtomEye [43].

\section{Results and discussion}

\subsection{Torsion deformation in twinned nanowires}

Figure 1a shows the variation of the applied torque as a function of the torsion angle $\theta$ during loading/unloading of a twinned nanowire with a diameter of $10 \mathrm{~nm}$ and twin spacing of $6.6 \mathrm{~nm}$. Yielding occurs at a small torsion angle $\left(\theta=17^{\circ}, \gamma_{\max }=0.033\right)$. The torsion stiffness of the twinned nanowire is $6.4 \times 10^{5} \mathrm{nN} \cdot \mathrm{nm}^{3}$, almost the same as that of pristine nanowires (Fig. A1). TBs have hence little effect on the shear modulus of the nanowire, a result consistent with a previous study [44]. As shown in Fig. 1a, after plastic yield, the nanowire first undergoes a strain hardening as the torque continuously increases with an increasing applied twist angle $\left(17^{\circ}<\theta<57.5^{\circ}, 0.033<\gamma_{\max }<0.112\right.$, stage A), while the torque saturates for higher twist angles $\left(57.5^{\circ}<\theta<100^{\circ}, 0.112<\gamma_{\max }<0.194\right.$, stage B). Strong fluctuations occur during this stage B. After unloading at the end of the strain-hardening stage A, the twinned nanowire fully recovers. On the contrary, deformation is only partially recovered unloading after entering stage B. As shown below, strain hardening is associated with strain recoverability, mild fluctuations, and homogeneous deformation patterns while the saturation stage is associated with non-recoverable deformation, wild fluctuations, and complex domain patterns. 


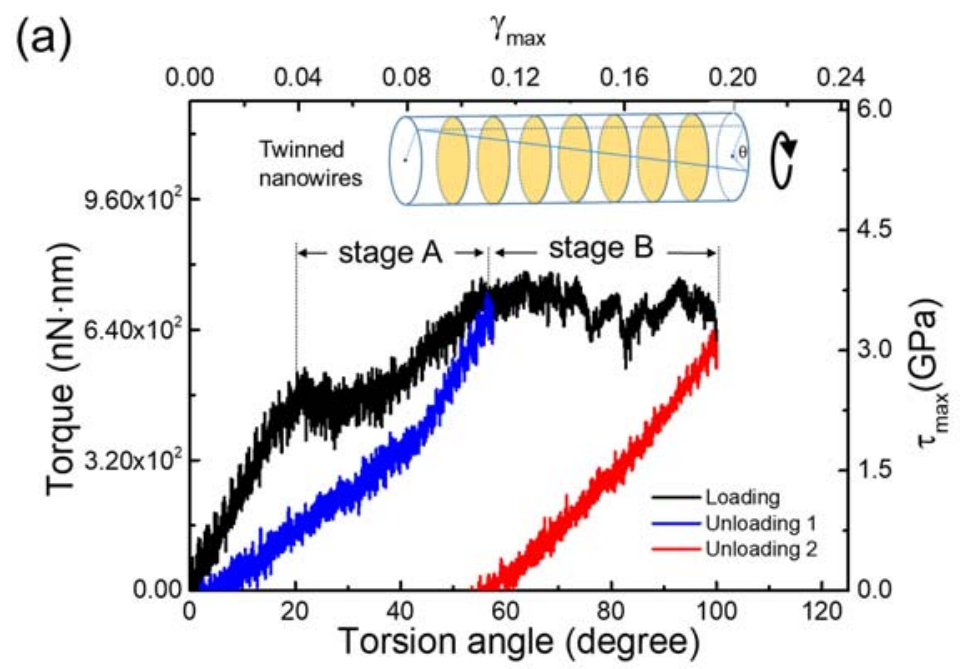

(b)

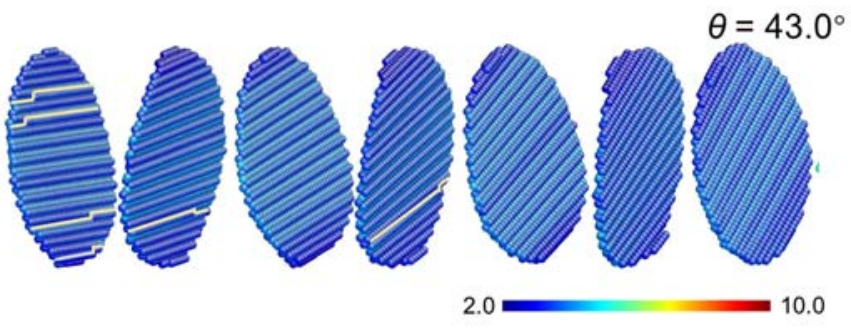

(c)

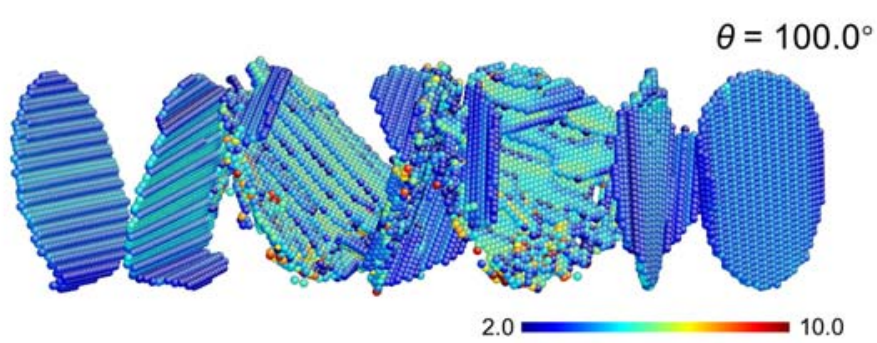

Fig. 1. Mechanical behavior of twinned nanowires under torsion in $\alpha-\mathrm{Fe}$, for a wire diameter $d$ of 10 $\mathrm{nm}$, a wire length $L$ of $44.8 \mathrm{~nm}$, and a TB spacing $s$ of $6.6 \mathrm{~nm}$. (a) Variation of torque (and $\tau_{\max }$ ) with the applied torsion angle (and $\gamma_{\max }$ ) for [112]-oriented twinned nanowires. Two stages of plasticity are distinguished according to the recoverability. Atomic configurations of TB at (b) $\theta=43^{\circ}$ and (c) $\theta=$ $100.0^{\circ}$. The colors are coded by the centrosymmetry parameter $P$ [45]. $P$ has the values of $2.0 \sim 3.0$ for atoms inside $\{112\}$ twin walls (dark blue), 3.0 4.5 for those around the $a / 6<111>$ partials (light blue), 4.5 6.0 for those at the non-conventional $\{110\}$ interfaces (green), and larger than 6.0 for atoms in heavily deformed regions (red).

Figures $1 \mathrm{~b}$ and $1 \mathrm{c}$ show the atomic configurations at two typical states after torsion of the twinned nanowires $\left(\theta=43^{\circ}, \gamma_{\max }=0.084\right.$ and $\left.\theta=100^{\circ}, \gamma_{\max }=0.194\right)$. We notice that the preexisting TBs undergo most of the deformation, while almost no dislocations are generated in the bulk between the TBs. Under weak applied twist $\left(\theta=43^{\circ}\right)$, the preexisting TBs form stripe patterns, while more complex patterns emerge when the twist angle is large $\left(\theta=100^{\circ}\right)$. The latter regime corresponds to stage B. Hence, the complexity of the microstructure is associated with different recoverability upon unloading: when unloading a twinned nanowire 
before the formation of complex patterns, the sample almost entirely recovers back to the original state (blue lines in Fig. 1a). Once complex patterns are formed, the shape recovery is largely suppressed and permanent deformation remains after unloading (red lines in Fig. 1a).

We further checked the evolution of the microstructure inside the twin walls under twisting. Figure 2 shows typical images of the structure of the interfaces at different stages of deformation. The left panel shows schematic illustrations of key features of the twin wall structure, the right panel shows the corresponding atomic images. Starting from an undeformed state with preexisting $\{112\}$ twin planes (Fig. 2a), we find that the initial twin planes transform to $\{110\}$ interfaces via the motion of $a / 6[11 \overline{1}]$ partial dislocations under small torsion (Fig. 2b). Specifically, partial dislocations nucleate at the surface and glide in the (112) plane, resulting in a lattice misfit in one (101) atomic layer. The sequential partial dislocations follow a similar path, i.e. they nucleate and propagate in the next (101) layer. A nonconventional $\{110\}$ interface is gradually formed by piling up $a / 6<111>$ partial dislocations on sequential (112) planes. Such $\{110\}$-type interfaces were reported in nanowires sustaining bending deformation [22]. A closer inspection reveals that the partial dislocations contain kinks. The kinks undergo some rotation under twisting. Considering the twinning partial as a primary "kink", the step in the partial dislocation line appears as a secondary kink and this twisted nanowire forms a nonconventional structure with walls inside walls and kinks inside kinks (Fig. 2b). This domain wall structure has been previously reported in ferroelectric domain walls [46, 47] but has never been reported in metallic nanowires. At larger rotation angles, in deformation stage B, the TBs deform by the collective motion of partial dislocations in different orientations, generating wild fluctuations and forming complex twin patterns (Fig. 2c). This movement generates intersections and junctions that are indicated by yellow arrows in Fig. 2c. 

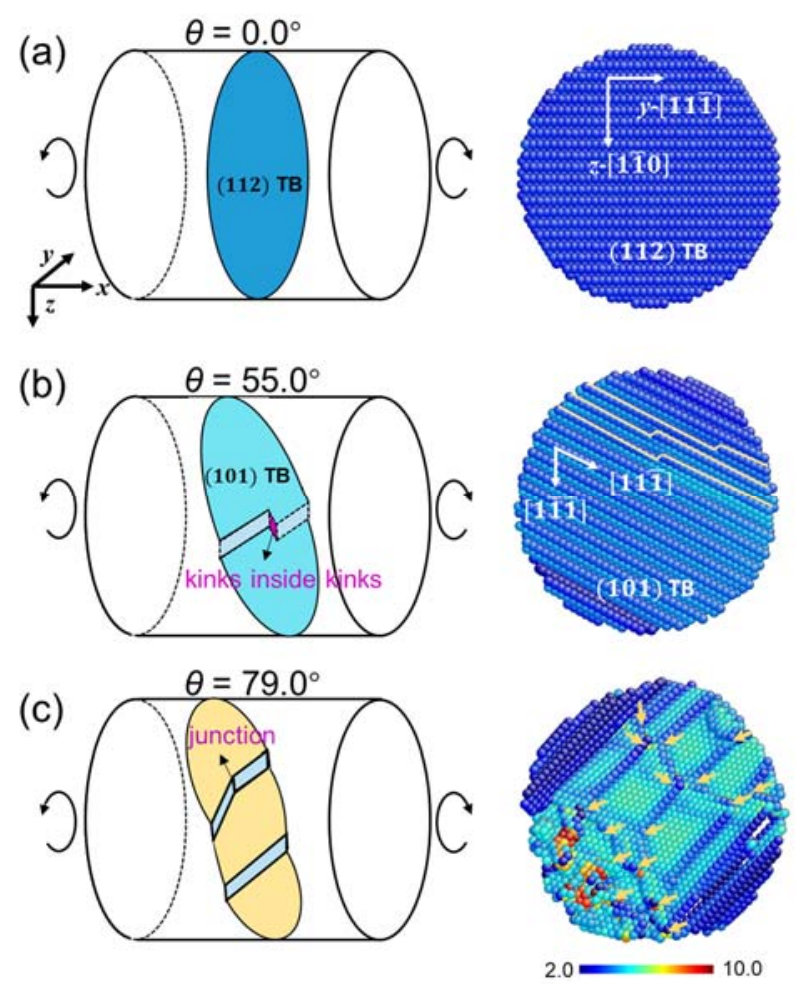

Fig. 2. (a) Initial configuration with conventional $\{112\}$ twin walls. (b) The $\{112\}$ twin walls transform to $\{110\}$ interfaces with parallel kinks motion. (c) Complex domain structures in highly twisted nanowires in the wild deformation regime. Kinks nucleate along various directions and form junctions (indicated by yellow arrows) in a highly deformed $\{110\}$ interfaces. The colors of atomic images are coded by the centrosymmetry parameter $P$ [45]. $P$ has the values of $2.0 \sim 3.0$ for atoms inside $\{112\}$ twin walls (dark blue), 3.0 4.5 for those around the $a / 6<111>$ partials (light blue), $4.5 \sim 6.0$ for those at the non-conventional $\{110\}$ interfaces (green), and larger than 6.0 for atoms in heavily deformed regions (red).

\subsection{Statistical analysis of the deformation process}

To characterize the deformation dynamics of TBs under torsion, we define the excess energy of the structural change (such as TB movements). The evolution of torque during deformation in the twinned sample is strongly 'jerky', which means that the stored strain energy is relaxed through discontinuous steps, in particular at large torsion angles. We quantify a 'jerk' in the following way: the potential energy $E_{\mathrm{p}}$ evolves discontinuously with increasing and decreasing torque. We square the first time derivative and define a jerk strength as $J \sim\left(\mathrm{d} E_{\mathrm{p}} / \mathrm{d} t\right)^{2}$, where $\mathrm{d} t$ is the timestep ( $5 \mathrm{fs}$ in our statistical analysis). This quantity is known as a slew rate in electrical engineering, plasma physics etc. [48]. Next, we carried out a statistical analysis of these energy fluctuations and correlate the strength distribution profile with the microstructural changes at each stage of deformation. 
Considering the situation depicted in Fig. 1, we first analyze deformation stage A at small torsion angles $\left(17^{\circ}<\theta<57.5^{\circ}\right.$ ) (Fig. 3a). The evolution of the torsion deformation is not smooth because TB movements are jerky. The probability density function (PDF) of jerks is peaked around a characteristic value of $9.1 \times 10^{-8} \mathrm{eV}^{2} \cdot \mathrm{fs}^{-2}$ (Fig. 3b). Within this deformation stage, jerks are related to nucleation and propagation of kinks. The peaked PDF signifies that kink nucleation and motion is associated with a characteristic energy scale, which is related to the motion of secondary kinks (Fig. 2b). The associated dynamics is mild [5].
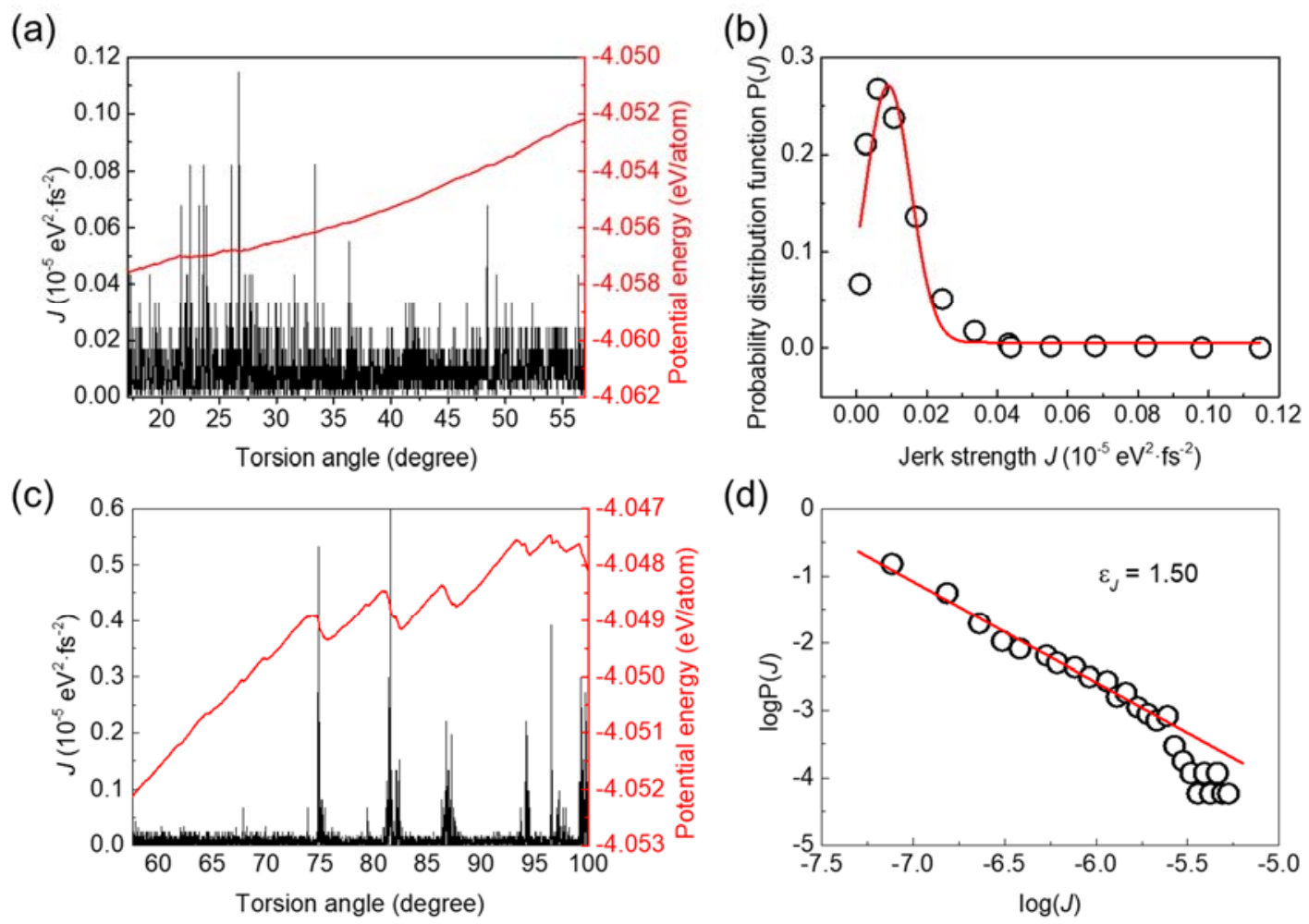

Fig. 3. Statistical analysis of jerks strength for twinned nanowires deformed under torsion $(d=10 \mathrm{~nm} ; L$ $=44.8 \mathrm{~nm} ; s=6.6 \mathrm{~nm}$ ). (a) Variation of the potential energy (red lines) with the torsion angle and the corresponding jerk spectrum (black lines) at stage A $\left(17^{\circ}<\theta<57.5^{\circ}\right)$. (b) The corresponding PDF of jerks strength. The red line is a fitted Gaussian function, where the maximum value occurs at $9.06 \times 10^{-}$ ${ }^{8} \mathrm{eV}^{2} \cdot \mathrm{fs}^{-2}$, and the standard deviation is $6.43 \times 10^{-8} \mathrm{eV}^{2} \cdot \mathrm{fs}^{-2}$. (c) Variation of potential energy (red line) and the corresponding jerk spectrum (black line) in stage $\mathrm{B}\left(57.5^{\circ}<\theta<100^{\circ}\right)$. (d) Log-log plot of the PDF of jerks strength showing a power-law distribution characterizing a wild regime.

At large torsion angles $\left(\theta>57.5^{\circ}\right)$, the twinned nanowire sustains large deformations through the motion of kinks and TBs. We choose the interval $57.5^{\circ}<\theta<100^{\circ}$ to determine the jerk spectra (Fig. 3c). Large torsion angles generate kinks in this regime. These kinks propagate along different directions, forming complex patterns including kink intersections and junctions. Consequently, TBs are highly deformed and hardly recognizable in Fig. 1c. Figure 3d shows that the PDF of jerks strength exhibits a power-law distribution, $P(J) \sim J^{-\varepsilon_{J}}$ with an exponent 
$\varepsilon J=1.50 \pm 0.05$. This implies a wild regime of deformation [5]. Various mechanisms can be associated with these wild fluctuations. By tracking the microstructural changes, we observed that many jerks are generated by depinning of jammed/entangled configurations within the TBs, while few large jerks are associated with the initiation of screw dislocations in the bulk (Fig. A2).

In summary, stage A is characterized by the prevalence of a characteristic jerk strength, while stage $B$ is associated with scale-free jerk statistics. One may therefore wonder if the transition from stage $\mathrm{A}$ to $\mathrm{B}$ could be signaled by a growing maximum jerk strength $J_{c}$ possibly diverging at a critical point. We checked this possibility as follows. For a power-law distribution with a cutoff, the distribution of probability of the jerks strength would be $P(J)=$ $J^{-\varepsilon_{J}} f\left(J / J_{\mathrm{c}}\right)$, where $\varepsilon_{J}$ is the exponent, $f(x)$ is a scaling function rapidly decaying for $x>1$, $J_{c}$ is the cutoff scale. We can use a dimensionless ratio $\left\langle J^{2}\right\rangle /\langle J\rangle^{2}$ to track the possible changes in the distribution $P(J)$. The heavier is the tail of the distribution, the larger will be this ratio.

It can be shown that this ratio scales as $J_{\mathrm{c}}{ }^{\left(\varepsilon_{J}-1\right)}$, hence we can monitor a possible evolution of the maximum event size $\left(\sim J_{c}\right)$ using $\left\langle J^{2}\right\rangle /\langle J\rangle^{2}$ during the whole torsion deformation. To obtain a statistically stable result, we calculate this ratio over rotation bins $\Delta \theta=2^{\circ}$ (each bin contains 8000 data points). Figure 4 shows the variation of $\left\langle J^{2}\right\rangle /\langle\rangle^{2}$ as a function of applied torsion angle $\theta$. We found that this ratio remains low, signing mild distributions (Fig. 3b) for $\theta<72^{\circ}$, without any detectable long-term increase as approaching the transition to stage $\mathrm{B}$, while this ratio strongly increases after that. The rough boundary separating two regimes at $\theta=72^{\circ}$ (the blue dotted line) is some kind of "delayed" (or "overdue") in comparison to that of $\theta=57.5^{\circ}$ determined above (the red dotted line). Thus, there is a crossover $\left(57.5^{\circ}<\theta<72^{\circ}\right)$ for regimes separated by statistical laws and maximum event size. This analysis indicates that thiscrossover from stage A to stage B is not a critical transition, where the maximum event size would increase as approaching the transition and diverge at this point. We found kinks with different orientations starting to nucleate and moving to the free surface in this crossover regime, where the generated jerky energy signals are still very weak. Before the crossover, only parallel kinks nucleate and propagate with small signals. The strong kinks pinning-depinning events occurs statistically with big signals after that. 


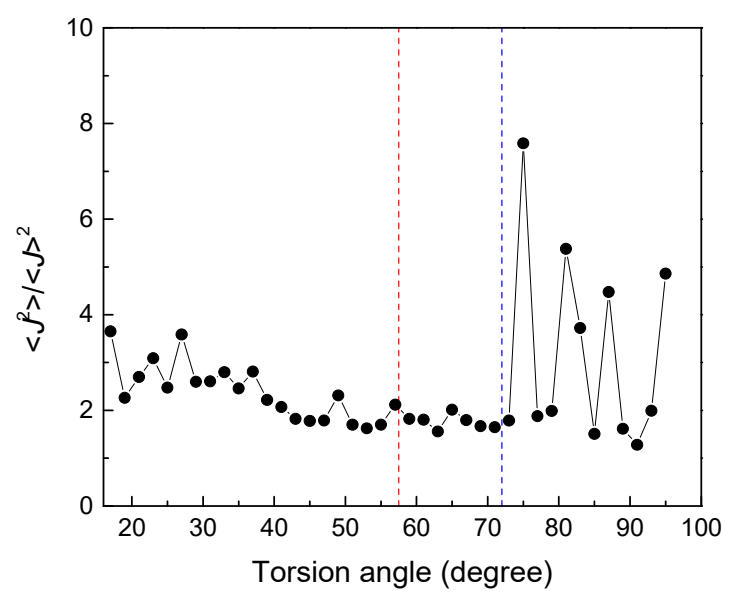

Fig. 4. The variation of ratio $\left\langle J^{2}\right\rangle /\langle\rangle^{2}$ as function of the torsion angle during torsion over successive windows of 8000 data points (corresponding to torsion angle of $2^{\circ}$ ) in a twinned nanowire ( $d=10 \mathrm{~nm} ; L$ $=44.8 \mathrm{~nm} ; s=6.6 \mathrm{~nm}) . J$ is the jerks strength and $\langle J\rangle$ is the jerks strength averaged over the time interval.

We calculated the degree of wildness, which is defined as $W=\frac{\sum_{J>J_{t} J_{i}}}{\sum_{\text {total }} J_{i}}[5,8] . J_{\mathrm{t}}$ is the jerk strength threshold. We used an open-source package to obtain the value of threshold $J_{\mathrm{t}}$ [49]. Specifically, the optimal threshold is determined by making a power-law fit of the complementary cumulative distribution function (CCDF) from each value in the dataset, and selecting the one that has the minimum Kolmogorov-Smirnov between the data and the fit. $J_{\mathrm{t}}$ is determined to be $9 \times 10^{-8} \mathrm{eV}^{2} \cdot \mathrm{fs}^{-2}$ in plasticity (see more details in Fig. A3). We checked that imposing a small change of $J_{\mathrm{t}}$ by a factor of $\pm 10 \%$ does not alter the exponents and degree of wildness significantly (see Fig. A4). For the Gaussian distribution, $J_{\mathrm{t}}$ is set to filter the long tail. We obtained a value of $W=0.06$ for stage A of deformation, and of $W=0.87$ for stage B at larger twist angles. This shows that mild and wild fluctuations can coexist during the torsion of twinned nanowires, much like during the dislocation-mediated plasticity of metallic materials, and confirms the transition from an essentially mild regime to an essentially wild regime when the applied torsion angle is increased.

We further explored the dynamics in the time domain. Figures $5 \mathrm{a}$ and $5 \mathrm{~b}$ show the PDF of waiting times between detected jerks in the mild and wild regimes, respectively. $R$, the ratio between the mean value and the standard deviation of waiting times, is used to quantify the intermittency of the process. The value of $R$ is close to 1.0 in the mild regime. Along with an exponential PDF (Fig. 5a), this implies an uncorrelated Poisson process without significant intermittency. Instead, at larger twist angles, the wild regime is associated with $R=0.82<1.0$ and a power law distribution of waiting times $\Delta t_{\mathrm{W}}$ (Fig. 5b), $P\left(\Delta t_{\mathrm{W}}\right) \sim \Delta t_{\mathrm{W}}{ }^{-\delta_{\mathrm{W}}}$ with $\delta \mathrm{w}_{\mathrm{W}}=1.68 \pm$ 0.08 , i.e., as expected, with intermittency and time correlations between events. 

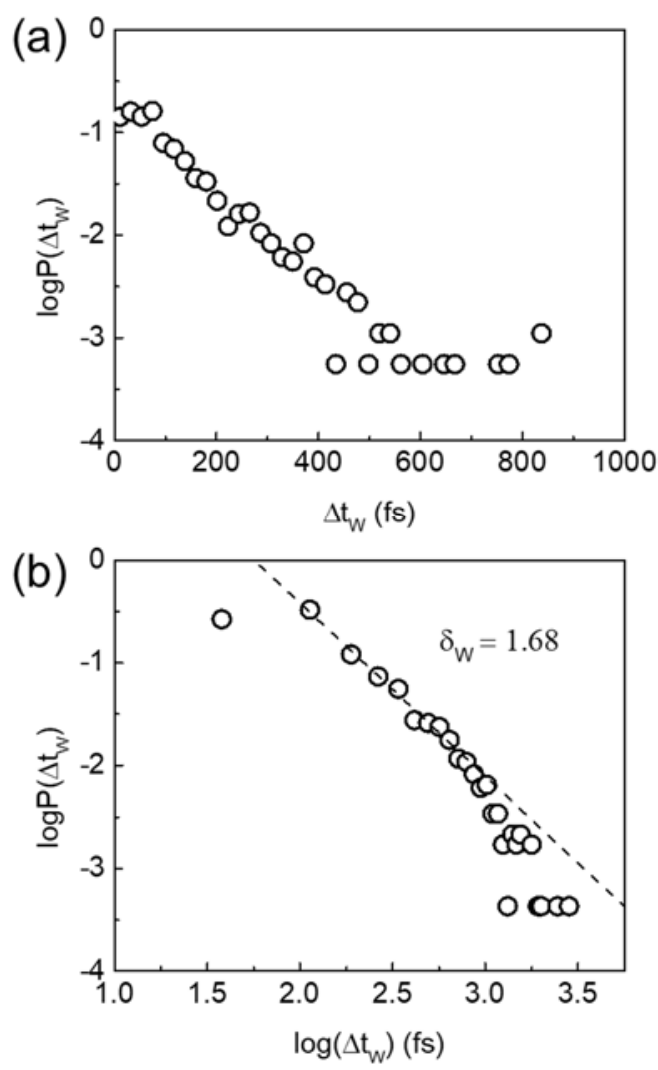

Fig. 5. Statistical analysis of waiting times between successive jerks for twinned nanowires deformed under torsion ( $d=10 \mathrm{~nm} ; L=44.8 \mathrm{~nm} ; s=6.6 \mathrm{~nm}$ ). The corresponding PDF shows (a) an exponential distribution for stage $\mathrm{A}\left(17^{\circ}<\theta<57.5^{\circ}\right)$ and (b) a power-law distribution for stage $\mathrm{B}\left(57.5^{\circ}<\theta<100^{\circ}\right)$.

The statistical results above were obtained by using the signals of jerks strength. We further carried out the statistical analysis on the signals of "true" avalanche energies, which were calculated as the integration of $-\mathrm{d} E_{\mathrm{p}} / \mathrm{d} t$ over the duration. Figure 6a shows the complementary cumulative distribution functions (CCDF) of avalanche energies in the wild regime. It follows a good power-law distribution. The exponent is 1.66, larger than that of 1.50 obtained using jerk strength signals in Fig. $3 \mathrm{~d}$. The CCDF of waiting times $\Delta t_{\mathrm{w}}$ and duration times $\Delta t_{\mathrm{D}}$ also show the power-law distributions, as shown in Fig. $6 \mathrm{~b}$ and $6 \mathrm{c}$. The exponents $\delta \mathrm{w}(=1.91)$ for waiting times and $\delta \mathrm{D}(=1.98)$ for duration times are very close, which keeps agreement with previous study [50]. 

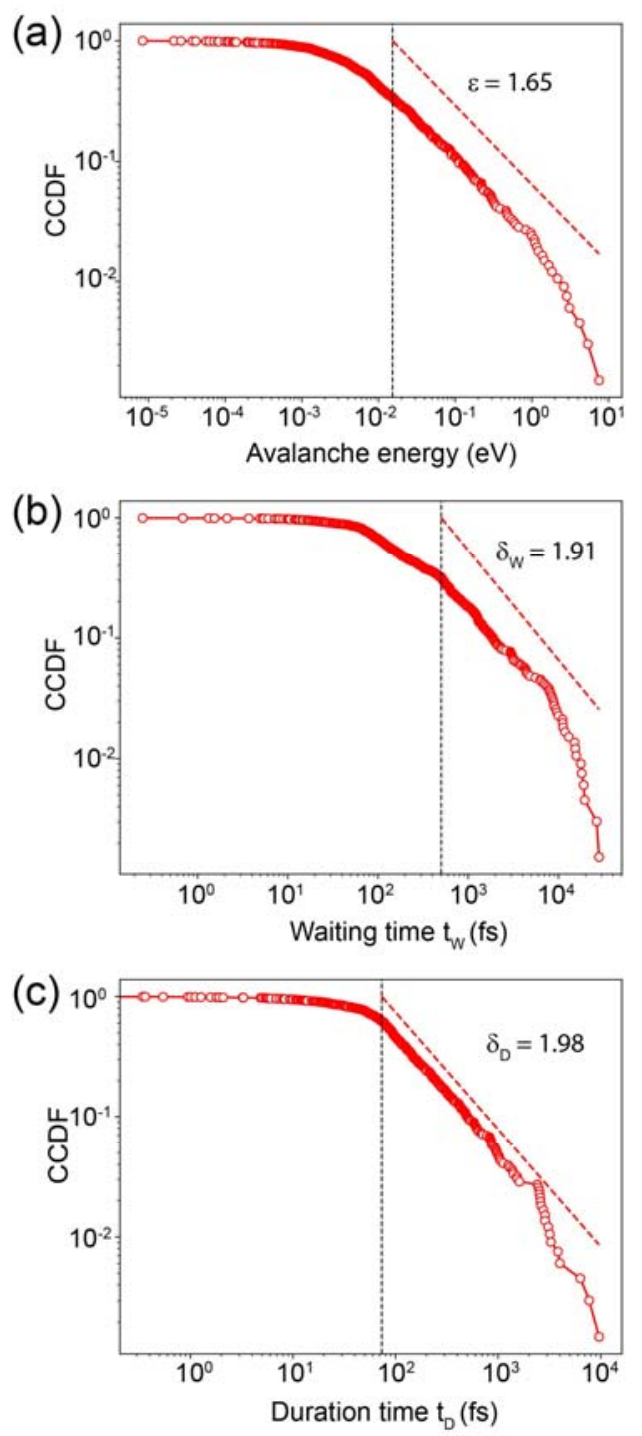

Fig. 6. Statistical analysis of (a) avalanche energies, (b) waiting times and (c) duration times for twinned nanowires in the wild regime $(d=10 \mathrm{~nm} ; L=44.8 \mathrm{~nm} ; s=6.6 \mathrm{~nm})$.

\subsection{Size effects}

The deformation dynamics of twisted nanowires with $d=10 \mathrm{~nm}, s=6.6 \mathrm{~nm}$ is given in section 3.2. We now extend this analysis to other configurations in order to investigate the effects of the sample size (wire diameter $d$ ) and of an internal length scale (the TB spacing $s$ ) on the statistical results. We first analyze the influence of the wire size on the torsion deformation with $d=5 \mathrm{~nm}, 10 \mathrm{~nm}$ and $15 \mathrm{~nm}$ while keeping the aspect ratio length/diameter at 4.5 (Fig. 7a). The twin boundary spacing is fixed at $6.6 \mathrm{~nm}$. The total number $N$ of TBs within the nanowire increases linearly with the diameter $N \sim d$. 
(a)
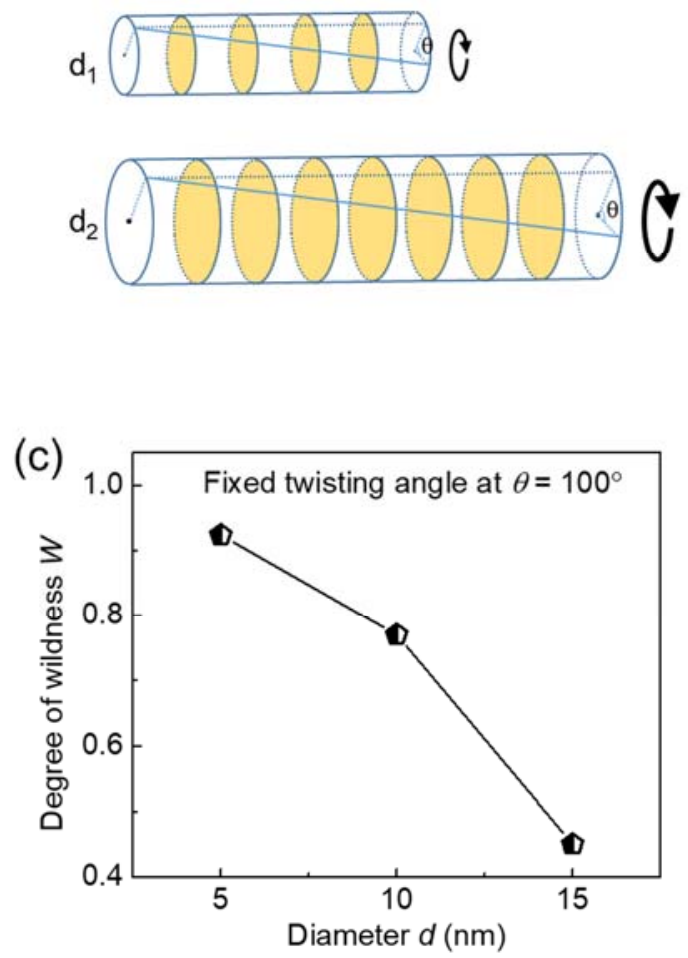

(b)

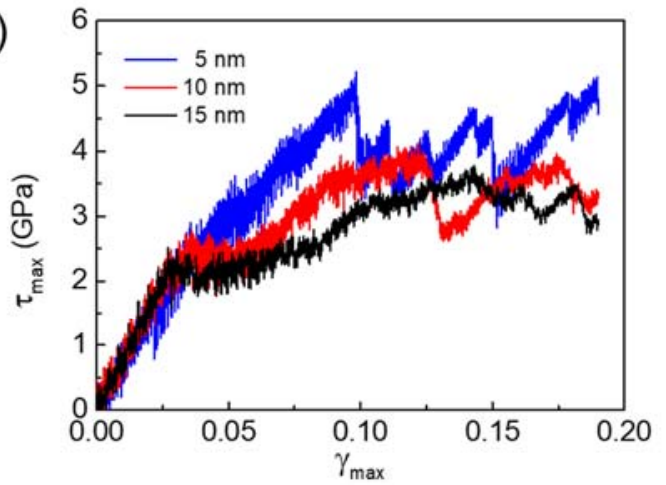

(d)

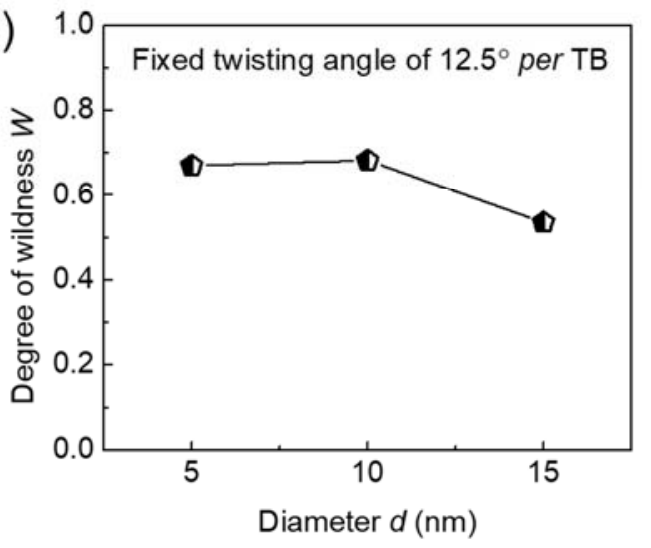

Fig. 7. The effect of external size (diameter $d$ ) on the torsion deformation of twinned nanowires. (a) Schematic illustration of the twinned structure by varying the wire diameter and keeping the aspect ratio fixed (b) Maximum shear stress $\tau_{\max } v s$ maximum shear strain $\gamma_{\max }$ curves. Dependence of the degree of wildness $W$ on the wire diameter $d(\mathrm{c})$ at a fixed twist angle $\theta=100^{\circ}$ and (d) at a fixed twist angle $12.5^{\circ}$ per TB. $W$ is calculated in the regime from the yielding point to the given twist angle.

Corresponding stress-strain curves are shown in Fig. 7b. Two types of size effects are readily apparent: (i) a "smaller is stronger" size effect on the maximum shear stress sustained during deformation, and (ii) a "smaller is wilder" size effect of the stress fluctuations after yield. The corresponding jerk spectra are shown in Fig. A5. When increasing the wire diameter, the onset of the wild regime (equivalent to stage B in Fig. 1a) is postponed to a larger applied shear strain. Consequently, when calculated over the entire deformation history up to a rotation of $100^{\circ}$ corresponding to a maximum shear strain of $\gamma_{\max } \approx 0.19$, the degree of wildness $W$ decreases with increasing sample size (blue line in Fig. 7c), quantifying the "smaller is wilder" size effect. Hence, in terms of external size effects, twinned $\alpha$-Fe nanowires deformed under torsion mimic the dislocation-mediated plasticity of metallic nanopillars which exhibit as well a "smaller is stronger" effect [51] as well as a "smaller is wilder" behavior [8]. However, as noted above, the configuration shown on Fig. 7a implies that $N \sim d$. Considering that nearly all the inelastic deformation occurs within the TBs [52], a larger diameter implies that the total strain will be shared among a larger number of TBs. Hence, for a fixed total rotation of $100^{\circ}$, each TB will 
deform on average by an angle $100^{\circ} / N$ (neglecting here possible boundary effects inducing less deformed TBs close to the wire ends). As the wildness increases with increasing deformation, when entering stage B (Fig. 1), this implies a decreasing $W$ under increasing $N$ and increasing $d$. Fig. $7 \mathrm{~d}$ shows the wildness calculated over the deformation history, up to a fixed rotation angle of $12.5^{\circ}$ per $T B$ The overall size effect on wildness is in this case very weak.

We then carried out another set of simulations by varying the internal TB spacing $s$, ranging from $3.8 \mathrm{~nm}$ to $15 \mathrm{~nm}$, while keeping the wire size at $d=10 \mathrm{~nm}$ (Fig. 8a). $s$ is inversely proportional to the TB density, $s \sim 1 / N$. An increase of number of TBs, i.e. a smaller $s$-value, implies a smaller maximum shear stress sustained during deformation (see Fig. 8b). The onset of the wild regime (equivalent to stage B in Fig. 1a) is postponed to larger applied shear strains (see the corresponding jerk spectra in Fig. A6). The postponement corresponds to the reduction of the twin spacing for higher TB densities. The plastic strain for a given applied rotation is carried by individual TBs and decreases with the increase of the TB density. Figure 9 shows the typical twin pattern with different $s$ at a fixed twist angle $\left(\theta=75^{\circ}\right)$. The twin patterns now become more complex with increasing $s$. Simultaneously, the samples with larger $s$ show a wilder plastic deformation. When then calculated the wildness over the entire torsion history up to $\theta=100^{\circ}$. The wildness $W$ decreases when decreasing the TB spacing $s$ (Fig. 8c). However, if one calculates the wildness up to a fixed rotation angle of $10.4^{\circ} \mathrm{per} T B$, the trend is the opposite: the wildness is largest for the smallest TB spacing $(3.8 \mathrm{~nm})$, then rapidly decreases to saturate at $W \approx 0.2$ for larger spacings (Fig. $8 \mathrm{~d}$ ). 
(a)
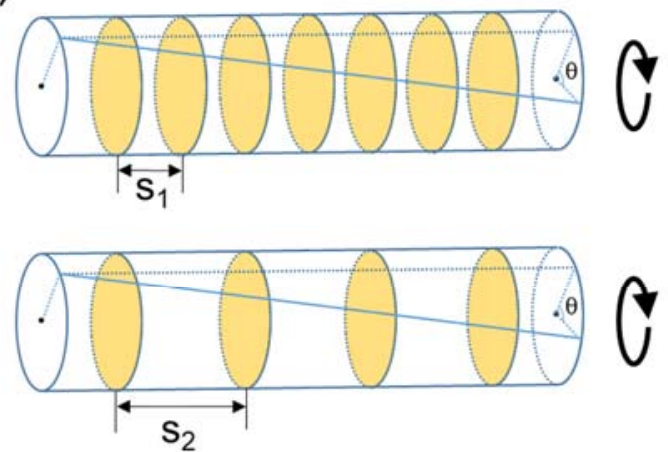

(c)

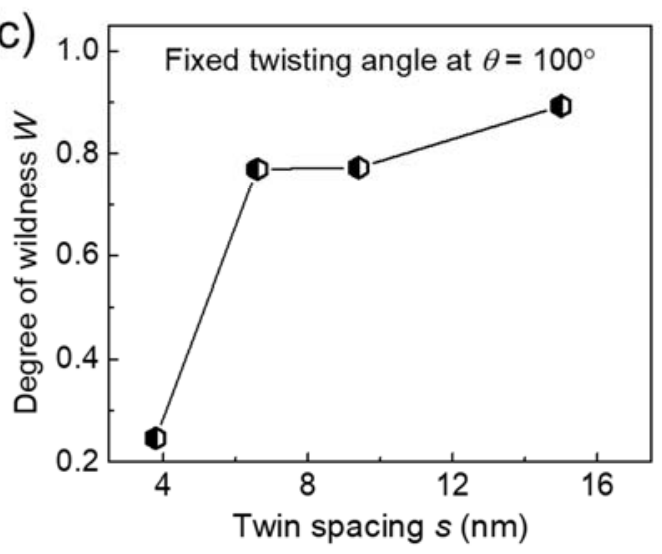

(b)

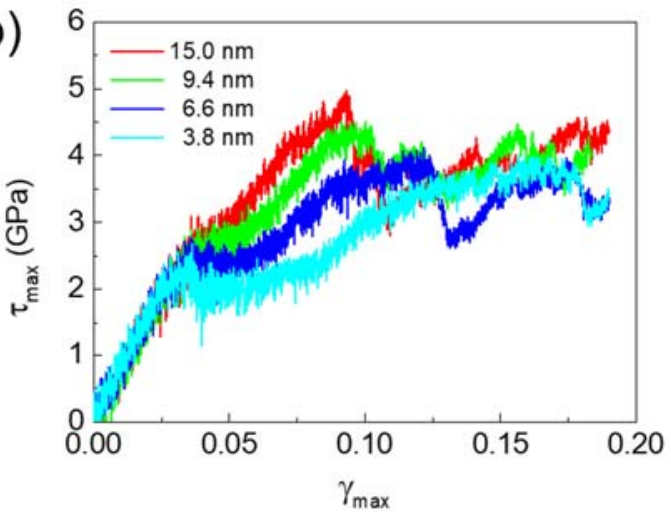

(d)

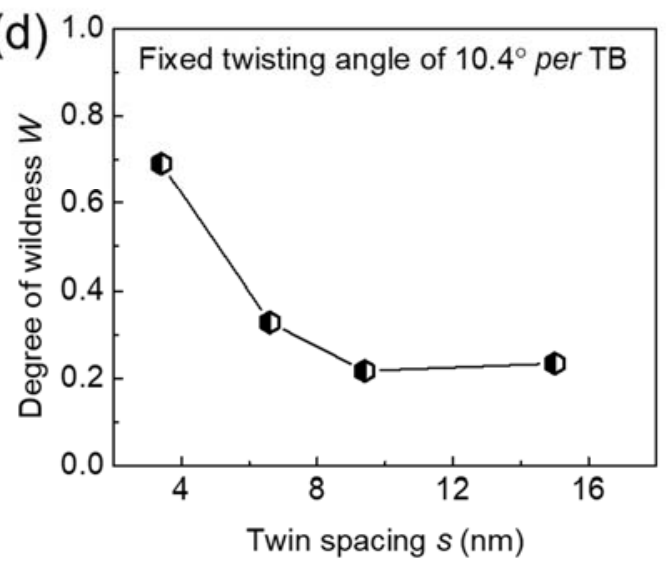

Fig. 8. The effect of internal size (TB spacing $s$ ) on the torsion deformation of twinned nanowires. (a) Schematic illustration of the twinned structure by varying the TB spacing and keeping the wire diameter fixed $(d=10 \mathrm{~nm})(\mathrm{b})$ Maximum shear stress $\tau_{\max } v s$ maximum shear strain $\gamma_{\max }$ curves. Dependence of the degree of wildness $W$ on the TB spacing $s$ (c) at a fixed twist angle $\theta=100^{\circ}$ and (d) at a fixed twist angle of $10.4^{\circ}$ per TB. $W$ is calculated in the regime from the yielding point to the given twist angle. 
(a)

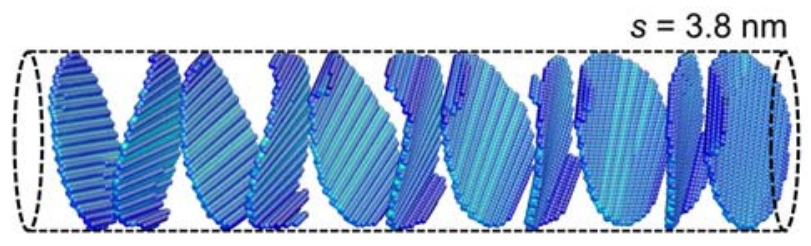

(b)
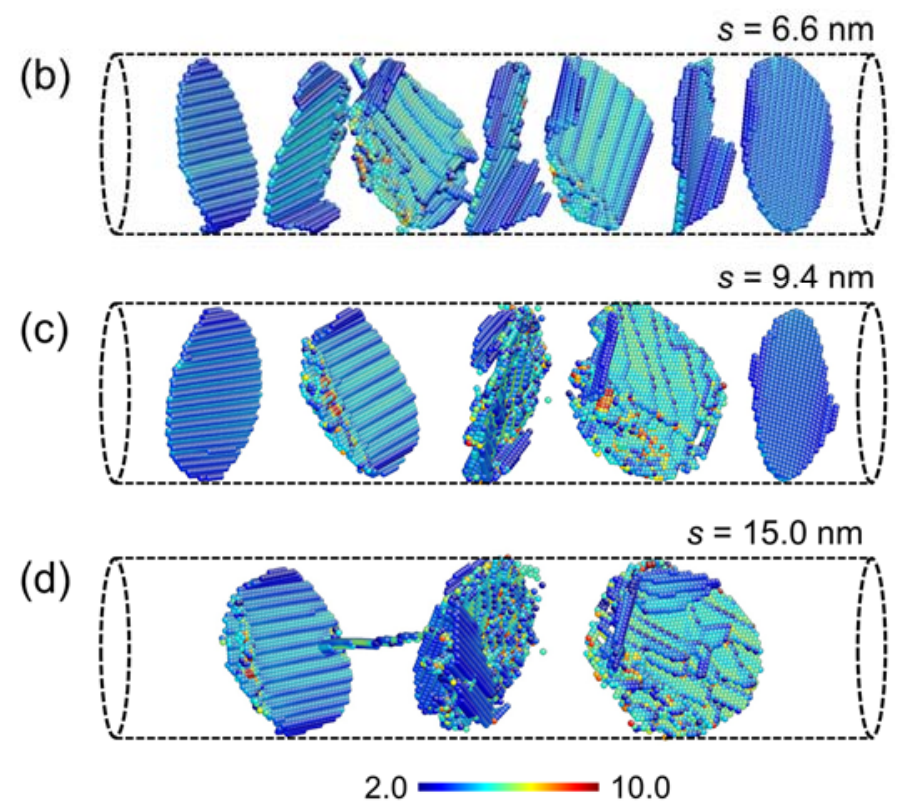

Fig. 9. Typical TB morphology at a given torsion angle $\left(\theta=75^{\circ}, \gamma_{\max }=0.145\right)$ for twinned $\alpha$-Fe nanowires with a twin spacing $s$ of (a) $3.8 \mathrm{~nm}$, (b) $6.6 \mathrm{~nm}$, (c) $9.4 \mathrm{~nm}$ and (d) $15.0 \mathrm{~nm}$. All samples have the same diameter $\sim 10 \mathrm{~nm}$ and length $\sim 44.8 \mathrm{~nm}$. The colors of atomic images are coded by the centrosymmetry parameter $P$ [45]. $P$ has the values of $2.0 \sim 3.0$ for atoms inside $\{112\} 10$ twin walls (dark blue), 3.0 4.5 for those around the $a / 6<111>$ partials (light blue), 4.5 6.0 for those at the nonconventional $\{110\}$ interfaces (green), and larger than 6.0 for atoms in heavily deformed regions (red).

\subsection{Tuning wildness by internal and external length scales}

The wildness of torsional deformation of the twinned nanowires is closely related to the complexity of the twin patterns, which can be characterized by the junction density $\rho$. Here $\rho$ is calculated as the number of junctions per unit volume over the entire nanowire. The number of junctions is counted for the same torsion angle $\left(\theta=100^{\circ}\right)$ for all samples. The circles in Fig. 10a show the variation of $W$ as a function of $\rho$ by collecting data from samples with different diameters and twin boundary spacings at a fixed twist angle. In addition, we collected the data points for a fixed twist angle per TB in Fig. 7d (triangle symbols in Fig. 10) and in Fig. 8d (square symbols in Fig. 10). A clear correlation between $\rho$ and $W$ is observed, namely an increase of the junction density implies wilder deformations. A similar relationship is obtained when $W$ is shown as function of the junction density per TB (Fig. 10b). This suggests that wild plastic fluctuations within twinned nanowires are associated with unlocking and rearrangements of complex patterns previously pinned by junctions between kinks (see Fig. 
2c). Such events involve the collective motion of multiple kinks along different directions, hence are much more energetic event than the individual motion of $a / 6<111>$ partial dislocations along a single direction, which characterizes the mild regime (Fig. 2b). An interesting contrast with dislocation-mediated plasticity within untwinned crystals occurs where dislocation junctions in multislip systems (such as FCC metals) are at the origin of dislocation entanglements, the formation of dislocation patterns, and consequently of isotropic strain hardening. Although possibly prone to episodic rearrangements, these strongly locked configurations are considered to frustrate the spreading of dislocation avalanches, hence to reduce wildness [8]. An opposite role is played by kink junctions in the present case (Fig. 10). During the pattern evolution, the individual twin walls (the big flat ones) first form kinks in the wall and do not interact. The nucleation and possible movement of these kinks gives some small jerks, corresponding to a mild plastic regime. Further torsion results in the generation of many kinks forming the more or less regular stripe patterns, still associated with a mild regime. Wild jerks arise when these kinks start colliding or interacting dynamically. As the torsion becomes even more severe, kinks, kinks inside kinks, and junctions are forming some very disordered kink configurations. The main interaction stems from the high energy of the junctions. The reorganization of such disordered configurations occurs through sudden events leading to wild plastic behavior. However, if the junctions were even denser, one might even switch back to a milder regime, reminiscent of dislocation tangles, which is not attained in our simulations and would need to be further explored. In this context, the junction density $\rho$ appears as an order parameter of the wild-to-mild transition. We finally note the absence of strain hardening in the wild regime of nanowires deformation (Figs 1a and 7b), which is fully consistent with the proposed scenario. The absence of strain hardening is also associated with wildness [53] in dislocation-mediated plasticity. 

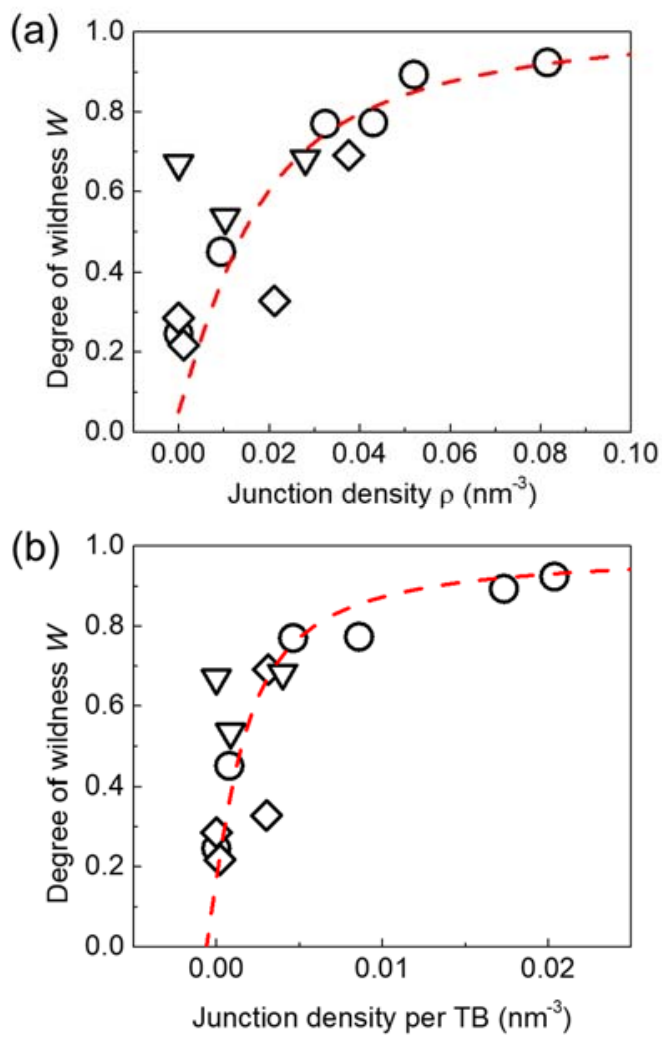

Fig. 10. The relationship between the degree of wildness and the junction density. The variation of $W$ as a function of (a) junction density $\rho$ and (b) junction density per TB. The circles indicate samples with different diameters and TB spacings at a fixed twist angle $\left(\theta=100^{\circ}\right)$. The triangles show samples with the same TB spacing but different diameters and a constant twist angle of $12.5^{\circ}$ per TB (Fig. 7d). The squares are samples with the same diameter but different TB spacing at a fixed twist angle of $10.4^{\circ}$ per TB (Fig. 8d). The red dashed lines are guides to the eyes.

The wire diameter and twin boundary density affect the junction density. As shown in Fig. 11, the junction density decreases when increasing the ratio $d / s$. Thus, we may tune the wildness either by controlling the external length scale $(d)$, the internal length scale $(s)$, or, more specifically, the ratio between both. This can be understood from the number $N$ of TBs within the nanowire: increasing $d$ or decreasing $s$ increases the number of TBs that share the total imposed deformation. They reduce the strain accommodated by each $\mathrm{TB}$, hence postpone the onset of stage $\mathrm{B}$ and reduce the wildness (Fig. 7c and 8c). On the contrary, by taking into account this sharing effect per $T B$ by calculating $W$ up to a fixed rotation angle, no significant sample size effect is observed over the range explored in Fig. $7 \mathrm{~d}$. The wildness only grows when the TB spacings fall below $5 \mathrm{~nm}$. This might indicate the onset of significant mechanical interactions between neighboring TBs. For close enough TBs, a kink deformation nucleating within one TB can nucleate other kinks in other TBs through elastic stress redistributions within the matrix, generating avalanches. Owing to the fast decay of these elastic interactions with 
distance [54, 55], such mechanism is expected to vanish for TB spacings above few nanometers (Fig. 8d). Dedicate simulations would be needed to confirm and quantify this point which is left for future work.

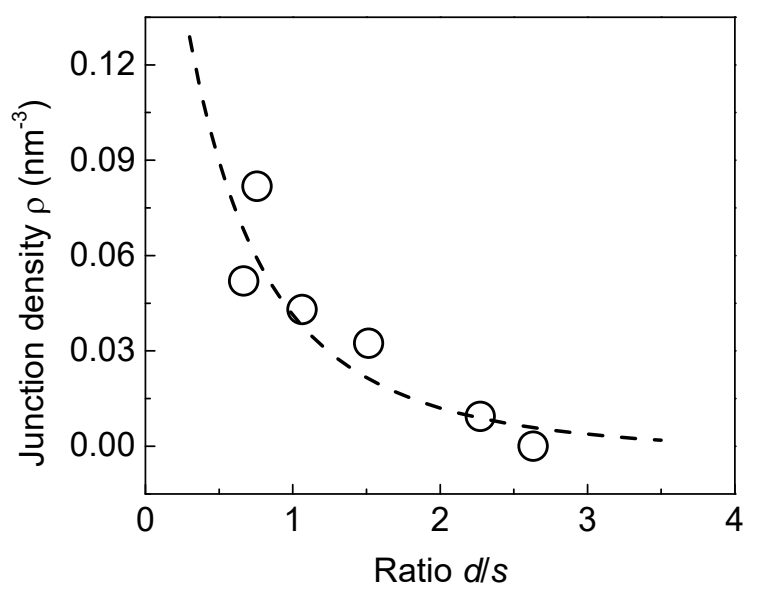

Fig. 11. The variation of the junction density as function of $d / s$, for all samples (all diameters, all TB spacings) at a constant twist angle $\theta=100^{\circ}$. The black dashed lines are guides for the eyes.

\section{Conclusion}

(a) Atomic simulations reveal the mechanism of torsion of pre-twinned $\alpha$-Fe nanowires. At small twist angles in deformation stage $A$, the preexisting $\{112\}$ twin planes progressively transform into $\{110\}$ interfaces through the motion of $a / 6<111>$ partial dislocations on sequential (112) planes. At larger twist angles at stage B, more complex twin patterns are formed with a high density of junctions when multiple kinks intersect.

(b) The deformed nanowires exhibit a good recoverability upon unloading from a simple twin pattern in stage A. The deformation can only partially recover once complex twin patterns are formed in stage $\mathrm{B}$.

(c) The dynamics of the deformation process shows characteristic statistical properties, depending on the deformation stage. The PDF of jerks strength is approximately Gaussian, associated with a characteristic strength scale, and non-intermittent at small torsion angles. This characterizes mild dynamics as previously observed experimentally by Faran et al $[46,47]$. For large torsion angles, kinks move along different directions, they form junctions and jammed configurations. The reorganization of such jammed configurations under increasing torsion 
occurs through intermittent avalanches where the PDF of jerks energies are power-law distributed. This defines wild dynamics.

(d) The torsion plasticity of twinned $\alpha$-Fe nanowires displays a transition from mild dynamics at small angles to wild dynamics at larger angles. The wildness is tightly linked to the junction density. It can be tuned by the external length scale (wire diameter) by keeping the twin boundary spacing constant, and/or by an internal length scale (twin boundary spacing) by keeping the diameter constant. The formation of kinks and junctions could act as the pinning sites to dislocations glide, leading to a pronounced hardening effect under mechanical deformation.

\section{Appendix A. Supplementary data}

See supplementary materials for more details of the torsion of $\alpha$-Fe nanowires.

\section{Acknowledgement}

X.D. and J.S. appreciate the support of NSFC (51931004, 51621063) and 111 project (BP2018008). E.K.H.S. is grateful to EPSRC (EP/K009702/1) and the Leverhulme Foundation (RPG-2012-564) for support. S.L. appreciates the support from the NKRDPC (Grant No. 2019YFA0307900). 


\section{ReferencesUncategorized References}

[1] R. Becker, E. Orowan, Sudden expansion of zinc crystals, Z. Phys 79 (1932) 566-572.

[2] J. Weiss, D. Marsan, Three-dimensional mapping of dislocation avalanches: clustering and space/time coupling, Science 299(5603) (2003) 89-92.

[3] J. Weiss, M.C. Miguel, Dislocation avalanche correlations, Materials Science and Engineering: A 387 (2004) 292-296.

[4] M.C. Miguel, A. Vespignani, S. Zapperi, J. Weiss, J.-R. Grasso, Intermittent dislocation flow in viscoplastic deformation, Nature 410(6829) (2001) 667.

[5] J. Weiss, W.B. Rhouma, T. Richeton, S. Dechanel, F. Louchet, L. Truskinovsky, From mild to wild fluctuations in crystal plasticity, Phys. Rev. Lett. 114(10) (2015) 105504.

[6] D.M. Dimiduk, C. Woodward, R. LeSar, M.D. Uchic, Scale-free intermittent flow in crystal plasticity, Science 312(5777) (2006) 1188-1190.

[7] S. Brinckmann, J.-Y. Kim, J.R. Greer, Fundamental differences in mechanical behavior between two types of crystals at the nanoscale, Phys. Rev. Lett. 100(15) (2008) 155502.

[8] P. Zhang, O.U. Salman, J. Zhang, G. Liu, J. Weiss, L. Truskinovsky, J. Sun, Taming intermittent plasticity at small scales, Acta Mater. 128 (2017) 351-364.

[9] F.F. Csikor, C. Motz, D. Weygand, M. Zaiser, S. Zapperi, Dislocation avalanches, strain bursts, and the problem of plastic forming at the micrometer scale, Science 318(5848) (2007) 251-254.

[10] T. Hu, L. Jiang, A. Mukherjee, J. Schoenung, E. Lavernia, Strategies to approach stabilized plasticity in metals with diminutive volume: a brief review, Crystals 6(8) (2016) 92.

[11] S. Papanikolaou, G. Po, The $\Lambda$-invariant and topological pathways to influence sub-micron strength and crystal plasticity, arXiv preprint arXiv:1906.01482 (2019).

[12] S. Papanikolaou, Y. Cui, N. Ghoniem, Avalanches and plastic flow in crystal plasticity: an overview, Modell. Simul. Mater. Sci. Eng. 26(1) (2018) 013001.

[13] T. Richeton, P. Dobron, F. Chmelik, J. Weiss, F. Louchet, On the critical character of plasticity in metallic single crystals, Mater. Sci. Eng., A 424(1-2) (2006) 190-195.

[14] E.K.H. Salje, H. Zhang, Domain boundary engineering, Phase Transit. 82(6) (2009) 452-469.

[15] C. Riek, D.V. Seletskiy, A.S. Moskalenko, J.F. Schmidt, P. Krauspe, S. Eckart, S. Eggert, G. Burkard, A. Leitenstorfer, Direct sampling of electric-field vacuum fluctuations, Science 350(6259) (2015) 420-423.

[16] E.K.H. Salje, S. Li, Z. Zhao, P. Gumbsch, X. Ding, Polar twin boundaries and nonconventional ferroelectric switching, Appl. Phys. Lett. 106(21) (2015) 212907.

[17] G. Lu, S. Li, X. Ding, E.K.H. Salje, Piezoelectricity and electrostriction in ferroelastic materials with polar twin boundaries and domain junctions, Appl. Phys. Lett. 114(20) (2019) 202901.

[18] E.K.H. Salje, S. Li, M. Stengel, P. Gumbsch, X. Ding, Flexoelectricity and the polarity of complex ferroelastic twin patterns, Phys. Rev. B 94(2) (2016) 024114.

[19] S. Li, X. Ding, J. Ren, X. Moya, J. Li, J. Sun, E.K.H. Salje, Strain-controlled thermal conductivity in ferroic twinned films, Sci. Rep. 4 (2014) 6375.

[20] X. Chen, L. Lu, K. Lu, Grain size dependence of tensile properties in ultrafine-grained Cu with nanoscale twins, Scripta Mater. 64(4) (2011) 311-314.

[21] S. Li, X. Ding, J. Deng, T. Lookman, J. Li, X. Ren, J. Sun, A. Saxena, Superelasticity in bcc nanowires by a reversible twinning mechanism, Phys. Rev. B 82(20) (2010) 205435.

[22] Y. Yang, S. Li, X. Ding, J. Sun, E.K.H. Salje, Interface Driven Pseudo - Elasticity in a - Fe Nanowires, Adv. Funct. Mater. 26(5) (2016) 760-767.

[23] L. Lu, R. Schwaiger, Z. Shan, M. Dao, K. Lu, S. Suresh, Nano-sized twins induce high rate sensitivity of flow 
stress in pure copper, Acta Mater. 53(7) (2005) 2169-2179.

[24] K. Otsuka, H. Sakamoto, K. Shimizu, A new type of pseudoelasticity in single variant twinned Martensites, Scripta Metall. 11(1) (1977) 41-46.

[25] K. Lu, Stabilizing nanostructures in metals using grain and twin boundary architectures, Nat. Rev. Mater.

1(5) (2016) 16019.

[26] L. Lu, Y. Shen, X. Chen, L. Qian, K. Lu, Ultrahigh strength and high electrical conductivity in copper, Science 304(5669) (2004) 422-426.

[27] X. He, S. Li, X. Ding, J. Sun, S.M. Selbach, E.K.H. Salje, The interaction between vacancies and twin walls, junctions, and kinks, and their mechanical properties in ferroelastic materials, Acta Mater. 178 (2019) 26-35.

[28] S. Van Aert, S. Turner, R. Delville, D. Schryvers, G. Van Tendeloo, E.K.H. Salje, Direct observation of ferrielectricity at ferroelastic domain boundaries in CaTiO3 by electron microscopy, Adv. Mater. 24(4) (2012) 523-527.

[29] A. Aird, E.K.H. Salje, Sheet superconductivity in twin walls: experimental evidence of, J. Phys.: Condens. Matter 10(22) (1998) L377.

[30] S. Li, X. Ding, J. Li, X. Ren, J. Sun, E. Ma, High-efficiency mechanical energy storage and retrieval using interfaces in nanowires, Nano Lett. 10(5) (2010) 1774-1779.

[31] S. Li, E.K.H. Salje, J. Sun, X. Ding, Large recovery of six-fold twinned nanowires of $\alpha$-Fe, Acta Mater. 125 (2017) 296-302.

[32] G. Sainath, B.K. Choudhary, T. Jayakumar, Molecular dynamics simulation studies on the size dependent tensile deformation and fracture behaviour of body centred cubic iron nanowires, Comput. Mater. Sci. 104 (2015) 76-83.

[33] G. Sainath, B.K. Choudhary, Deformation behaviour of body centered cubic iron nanopillars containing coherent twin boundaries, Philos. Mag. 96(32-34) (2016) 3502-3523.

[34] A.F. da Fonseca, D.S. Galvão, Mechanical properties of nanosprings, Phys. Rev. Lett. 92(17) (2004) 175502. [35] S. Evoy, D.W. Carr, L. Sekaric, A. Olkhovets, J.M. Parpia, H.G. Craighead, Nanofabrication and electrostatic operation of single-crystal silicon paddle oscillators, J. Appl. Phys. 86(11) (1999) 6072-6077.

[36] J. Hsieh, W. Fang, A novel microelectrostatic torsional actuator, Sens. Actuators A Phys. 79(1) (2000) 64-70. [37] P. Zhang, J.-J. Bian, J.-Y. Zhang, G. Liu, J. Weiss, J. Sun, Plate-like precipitate effects on plasticity of Al-Cu alloys at micrometer to sub-micrometer scales, Mater. Des. 188 (2020) 108444.

[38] M.S. Daw, M.I. Baskes, Embedded-atom method: Derivation and application to impurities, surfaces, and other defects in metals, Phys. Rev. B 29(12) (1984) 6443.

[39] M.I. Mendelev, S. Han, D.J. Srolovitz, G.J. Ackland, D. Sun, M. Asta, Development of new interatomic potentials appropriate for crystalline and liquid iron, Philos. Mag. 83(35) (2003) 3977-3994.

[40] S. Nose, A Unified Formulation of the Constant Temperature Molecular-Dynamics Methods, J. Chem. Phys. 81(1) (1984) 511-519.

[41] W.G. Hoover, Canonical Dynamics - Equilibrium Phase-Space Distributions, Phys. Rev. A 31(3) (1985) 16951697.

[42] S. Plimpton, Fast Parallel Algorithms for Short-Range Molecular-Dynamics, J. Comput. Phys. 117(1) (1995) 1-19.

[43] J. Li, AtomEye: an efficient atomistic configuration viewer, Modell. Simul. Mater. Sci. Eng. 11(2) (2003) 173. [44] T. Klöffel, E. Bitzek, B. Meyer, Impact of twin boundaries on bulk elastic constants: Density-functional theory data for Young's modulus of Ag, Data Brief 3 (2015) 209-215.

[45] C.L. Kelchner, S.J. Plimpton, J.C. Hamilton, Dislocation nucleation and defect structure during surface indentation, Phys. Rev. B 58(17) (1998) 11085. 
[46] E.K.H. Salje, O. Aktas, M.A. Carpenter, V.V. Laguta, J.F. Scott, Domains within domains and walls within walls: evidence for polar domains in cryogenic SrTiO 3, Phys. Rev. Lett. 111(24) (2013) 247603.

[47] E. Faran, E.K.H. Salje, D. Shilo, The exploration of the effect of microstructure on crackling noise systems, Appl. Phys. Lett. 107(7) (2015) 071902.

[48] F. Liu, G. Huang, B. Ganguly, Plasma excitation dependence on voltage slew rates in 10-200 Torr argonnitrogen gas mixture DBD, Plasma Sources Sci. Technol. 19(4) (2010) 045017.

[49] J. Alstott, E. Bullmore, D. Plenz, powerlaw: a Python package for analysis of heavy-tailed distributions, PloS one 9(1) (2014) e85777.

[50] S. Janićević, L. Laurson, K.J. Måløy, S. Santucci, M.J. Alava, Interevent correlations from avalanches hiding below the detection threshold, Phys. Rev. Lett. 117(23) (2016) 230601.

[51] M.D. Uchic, D.M. Dimiduk, J.N. Florando, W.D. Nix, Sample dimensions influence strength and crystal plasticity, Science 305(5686) (2004) 986-989.

[52] J. Wang, Z. Zeng, C.R. Weinberger, Z. Zhang, T. Zhu, S.X. Mao, In situ atomic-scale observation of twinningdominated deformation in nanoscale body-centred cubic tungsten, Nat. Mater. 14(6) (2015) 594.

[53] J. Weiss, Ice: the paradigm of wild plasticity, Philos. Trans. R. Soc. A 377(2146) (2019) 20180260.

[54] A.S. Everhardt, S. Damerio, J.A. Zorn, S. Zhou, N. Domingo, G. Catalan, E.K.H. Salje, L.-Q. Chen, B. Noheda, Periodicity-doubling cascades: Direct observation in ferroelastic materials, Phys. Rev. Lett. 123(8) (2019) 087603.

[55] G. Lu, S. Li, X. Ding, J. Sun, E.K.H. Salje, Electrically driven ferroelastic domain walls, domain wall interactions, and moving needle domains, Phys. Rev. Mater. 3(11) (2019) 114405. 